\title{
Locomotor Microactivities Associated with Therapeutic Responses in Patients with Seasonal Affective Disorders
}

\author{
Kyoko Ohashia, b Yoshiharu Yamamotoc Martin H. Teicher ${ }^{\mathrm{a}, \mathrm{b}}$ \\ a Developmental Biopsychiatry Research Program, McLean Hospital, Belmont, Mass., and \\ ${ }^{b}$ Department of Psychiatry, Harvard Medical School, Boston, Mass., USA; ${ }^{\circ}$ Educational \\ Physiology Laboratory, Graduate School of Education, The University of Tokyo, Tokyo, Japan
}

\section{Key Words}

Seasonal affective disorders · Locomotor activity · Bright light phototherapy · Wavelets .

Nonlinear dynamics . Psychomotor retardation

\section{Abstract}

Background: Psychomotor retardation, leaden paralysis and fatigue are often used to describe patients with depressive disorders. However, there is limited understanding of their meaning and how they are objectively manifested in the physical world. Patients with seasonal affective disorder (SAD) are characteristically hypoactive and experience restoration of energy during effective treatment with bright light. In this study, we attempt to identify quantitative metrics of psychomotor activity that correspond to the clinical perceptions of hypoactivity and to the early activating effects of treatment. Methods: Novel means of assessing the microstructure of activity was employed using wavelets and Hurst exponents to indicate the proclivity of subjects to persist at higher and lower levels of activity. This was assessed using actigraphs in 16 unmedicated patients with SAD before and following 2 weeks of bright light therapy. Results: Two weeks of phototherapy had no significant effect on mean levels of diurnal activity, but altered the microstructure of the activity. Specifically, phototherapy produced a significant reduction in inertial resistance in patients who had a $\geq 50 \%$ reduction in the Hamilton Depression Scale scores $(n=8)$, as reflected in a reduced tendency to persist at low levels of activity. There was also a strong correlation between ratings of fatigue and measures of persistence at high versus low activity in initial responders, but not in initial nonresponders. Conclusion: These findings suggest that light therapy alters the nature of diurnal activity troughs in early responsive patients, reducing their tendency to persist at low levels, possibly reflecting an alleviation of psychomotor retardation. 


\section{Background}

Seasonal affective disorder (SAD) is a recurring mood disorder that affects about $6 \%$ of adults living far from the equator [1]. Most patients with SAD have atypical depressive symptoms characterized by fatigue, hypersomnia, and carbohydrate cravings [1]. Objective measures of locomotor activity levels with wrist-worn actigraphs have reported that adults with SAD were, on average, 14\% less active than healthy controls [2]. However, there was considerable overlap in activity levels between groups despite prominent symptoms of fatigue or psychomotor retardation in subjects with SAD [2]. Traditional measures such as mean activity levels ignore the richness and complexity of human activity and potentially provide an incomplete picture of activity disturbances that we describe as psychomotor agitation or retardation and lack the sensitivity to detect therapeutic effects. In the present paper, we illustrate a new analysis of locomotor activities and show that this method reveals effects of treatment that were not discernible using conventional methods.

Locomotor activity patterns of humans are characterized by a prominent circadian cycle and often a modest 2-cycle-per-day hemicircadian component. Together, these rhythms account for about $50 \%$ of the variance. Otherwise, the pattern is essentially aperiodic [3]. A number of investigators have studied the time correlations of locomotor activity to assess hidden temporal characteristics [4]. These studies have shown that human physical activity has so-called scale-invariant features. This means that the pattern of fluctuations in activity has a fractal-like nature and appears similar whether one examines slow fluctuations over long periods of activity or rapid fluctuations during brief periods. Due to this self-similarity, it turns out that a single mathematical parameter called a 'power-law scaling exponent' essentially characterizes the pattern of fluctuations. Furthermore, this exponent characterizes the degree of persistency of activity, i.e., whether high periods of activity tend to be followed by high periods (persistent) or by low periods (antipersistent). This parameter conveys a great deal of information about the temporal structure of activity and may provide greater insight into the nature of activity disturbances in psychiatric disorders.

Ohashi et al. [5] reported that locomotor activity in children with SAD has different power-law scaling exponents compared to that of the age-matched controls. Another study with healthy adults found that the power-law scaling exponents were different for periods with higher (e.g., in motion) and lower (e.g., resting) activity levels [6]. Healthy controls had more antipersistent patterns of activity at higher than at lower levels of activity. This means that periods of high activity were associated with abrupt onsets and offsets, while periods of lower activity were associated with more gradual transitions. Patients with chronic fatigue syndrome [7] lacked these abrupt antipersistent bursts of activity and had equivalent scaling exponents during periods with higher and lower activity levels, possibly due to exaggerated fatigue [8]. Differentiating the scaling exponents for higher and lower activity levels improved sensitivities to evaluate the disease severity [9] and the effect of potential treatment [10] in patients with Parkinson's disease.

Bright light therapy has been reported to alleviate symptoms of SAD $[1,11]$. Previous studies quantifying the effect of bright light therapy have focused on circadian rest-activity rhythms [12]. However, little is known about the effects of bright light therapy activity patterns within the daytime period. In this study, we used the method incorporating bidirectional analysis of scaling exponents $[5,6,8]$ and studied the alterations in the time correlations of daytime locomotor activity data before and during the second week of bright light therapy in patients with SAD. Locomotor microactivities were examined separately for SAD patients responsive or nonresponsive to therapy as assessed using the 21-item Hamilton Depression Scale, with an 8-item addendum of atypical depressive symptoms for SAD (SIGHSAD) [13]. 


\section{Patients and Methods}

\section{Subjects}

Adult subjects were recruited by advertisement looking for individuals who met the criteria for SAD and were interested in a trial of bright light phototherapy. Subjects were assessed by the structured clinical interview (SCID-CV) [14] and met DSM-IV criteria for major depression with a seasonal pattern and Rosenthal-NIMH criteria for SAD [1]. The sample consisted of 12 women and 4 men who ranged in age from 21 to 52 years (mean \pm SD, $39.6 \pm 8.45$ years). All the subjects were studied during their natural daily routine and were free from psychotropic medications. The protocol was approved by the McLean Hospital Institutional Review Board, and all subjects provided informed written consent.

\section{Procedures}

Activity data were collected with an ambulatory activity monitor (Mini-Motionlogger Ambulatory Monitors Inc., Ardsley, N.Y., USA) that was worn on the nondominant wrist. The device counted all accelerations ( $>0.01 \mathrm{~g}$ force zero-crossing mode) and stored results in 1-min epochs. The subjects were instructed to wear the device continuously except while bathing and to record times when the device was taken off along with bedtimes and rise times. At least $72 \mathrm{~h}$ of continuous recording were collected during pretreatment (baseline), and again during the first and second weeks of phototherapy. Subjects received an open trial of bright light phototherapy using a tilted UV-filtered commercial light box (SunRay II, SunBox, Inc., Gaithersburg, Md., USA), which provided 10,000 lx of light at a distance of $58 \mathrm{~cm}$ from the source. Intensity was confirmed using a Minolta Chroma Meter II. Subjects received 30 min of daily treatment each morning (increasing to $60 \mathrm{~min}$ in nonresponders) using procedures described by Terman et al. [11]. Response to treatment was assessed weekly using the SIGH-SAD.

\section{Data Analyses}

We analyzed the daytime locomotor activities during 1 week prior to the bright light therapy as the baseline. Activity data were also collected during the second week of phototherapy (days 8-14) to examine the changes due to the treatment. Subjects continued to receive 30-60 min of bright light therapy in the morning during this phase.

Periods when the subjects removed the actigraph or napped were marked as invalid and interpolated linearly. Such periods were determined by the subjects' written records and Cole's algorithm for sleep detection [15]. Days containing less than 720 points of valid daytime data (i.e., less than $12 \mathrm{~h}$ ) were excluded from the analysis.

After the week of pretherapy activity recording, SIGH-SAD was administered to obtain a baseline depression score. The SIGH-SAD was again administered at the beginning and the end of the second week of therapy, and the scores were averaged for each subject to provide a depression score during treatment. For 3 subjects who lacked one of the post-treatment SIGH-SAD ratings, the single available score was used. We classified the patients into responders and nonresponders to compare the effect of therapy on locomotor activities. We defined 'responders' as those who showed $\geq 50 \%$ decreases in SIGH-SAD scores during light treatment compared to the pretherapy values, with the remaining patients defined as 'nonresponders'. Consequently, 8 patients ( 1 male/ 7 female) were categorized into the responder group, and the remaining 8 patients ( 3 male/5 female) were categorized into the nonresponder group. This resulted in marked decreases in the SIGH-SAD scores for the responders and in minimal change for the nonresponders. The two groups had nearly equivalent pretreatment SIGH-SAD ratings (table 1). 
Table 1. SIGH-SAD scores and average scaling exponents at maxima and minima

\begin{tabular}{|c|c|c|c|c|c|c|c|c|c|c|}
\hline & \multicolumn{5}{|c|}{ Before treatment } & \multicolumn{5}{|c|}{ Following treatment } \\
\hline & $\begin{array}{l}\text { HAMD } \\
\text { score }\end{array}$ & $\begin{array}{l}\text { retar- } \\
\text { dation }^{\text {a }}\end{array}$ & $\begin{array}{l}\text { fati- } \\
\text { gability }^{\mathrm{b}}\end{array}$ & maxima & minima & $\begin{array}{l}\text { HAMD } \\
\text { score }\end{array}$ & $\begin{array}{l}\text { retar- } \\
\text { dation }^{\text {a }}\end{array}$ & $\begin{array}{l}\text { fati- } \\
\text { gability }^{\mathrm{b}}\end{array}$ & maxima & minima \\
\hline \multicolumn{11}{|c|}{ Responder } \\
\hline Subj. 1 & 33 & 2 & 3 & 1.082 & 1.019 & 5 & 0 & 0 & 1.035 & 1.020 \\
\hline Subj. 2 & 33 & 0 & 3 & 1.019 & 1.025 & 1 & 0 & 0 & 1.001 & 0.940 \\
\hline Subj. 3 & 32 & 0 & 3 & 1.022 & 1.004 & 1 & 0 & 0 & 1.806 & 0.827 \\
\hline Subj. 4 & 24 & 0 & 2 & 0.796 & 0.918 & 11.5 & 0 & 1.5 & 0.883 & 0.902 \\
\hline Subj. 5 & 27 & 2 & 2 & 0.896 & 1.032 & 12.5 & 0.5 & 1.5 & 0.844 & 0.909 \\
\hline Subj. 6 & 27 & 2 & 2 & 0.856 & 0.876 & 2 & 0 & 1 & 0.852 & 0.870 \\
\hline Subj. 7 & 18 & 1 & 1 & 0.784 & 1.136 & 5 & 0 & 0.5 & 0.897 & 0.951 \\
\hline Subj. 8 & 37 & 1 & 3 & 0.957 & 0.857 & 15.5 & 0.5 & 1 & 0.848 & 0.768 \\
\hline Mean & 28.9 & 1.0 & 2.4 & 0.926 & 0.983 & 6.7 & 0.1 & 0.7 & 0.896 & 0.898 \\
\hline $95 \% \mathrm{CI}$ & $23.8-34.0$ & $0.2-1.8$ & $1.8-3.0$ & $0.834-1.019$ & $0.905-1.061$ & $1.9-11.4$ & $-0.1-0.3$ & $0.1-1.2$ & $0.828-0.963$ & $0.833-0.963$ \\
\hline \multicolumn{11}{|c|}{ Nonresponder } \\
\hline Subj. 9 & 32 & 2 & 4 & 0.826 & 0.677 & 26.5 & 0.5 & 2 & 0.947 & 1.000 \\
\hline Subj. 10 & 27 & 2 & 2 & 0.701 & 0.688 & 20 & 2 & 2 & 0.808 & 0.866 \\
\hline Subj. 11 & 32 & 2 & 2 & 1.116 & 1.131 & 26 & 2 & 2 & 0.877 & 0.978 \\
\hline Subj. 12 & 28 & 2 & 3 & 1.030 & 1.024 & 16 & 1 & 1.5 & 1.003 & 1.083 \\
\hline Subj. 13 & 24 & 1 & 2 & 0.721 & 0.838 & 27 & 0.5 & 2 & 0.850 & 0.949 \\
\hline Subj. 14 & 19 & 0 & 3 & 0.877 & 1.036 & 18 & 0 & 3 & 0.809 & 0.775 \\
\hline Subj. 15 & 38 & 2 & 4 & 0.991 & 0.985 & 32.5 & 1 & 3 & 0.881 & 0.962 \\
\hline Subj. 16 & 25 & 2 & 3 & 0.768 & 0.952 & 18.5 & 1.5 & 2.5 & 0.727 & 0.768 \\
\hline Mean & 28.1 & 1.6 & 2.9 & 0.879 & 0.916 & 23.1 & 1.1 & 2.3 & 0.863 & 0.923 \\
\hline $95 \% \mathrm{CI}$ & $23.2-33.0$ & $1.0-2.2$ & $2.2-3.6$ & $0.751-1.006$ & $0.777-1.055$ & $18.3-27.9$ & $0.5-1.7$ & $1.8-2.7$ & $0.791-0.935$ & $0.830-1.015$ \\
\hline
\end{tabular}

\section{Microstructure Analysis}

The scale-invariant dynamics of the locomotor activity data were examined by a modification $[6,8]$ of the wavelet transform modulus maxima (WTMM) approach [16]. The WTMM approach uses wavelets to detect discontinuities or singularities in the actigraph data. The basic idea behind the analysis is the following: the actigraph time series for each subject is matched to a series of wavelets that resemble right-side up or upside-down 'Mexican hats' (fig. 1a). The wavelets range in scale from 'skinny' to 'fat', reflecting the entire range of shortterm (rapid) to long-term (slow) fluctuations in activity. These wavelets are essentially templates for low-high-low activity patterns (fig. 1a, right green) or high-low-high activity level patterns (fig. 1a, left green) depending on the direction of the wavelet. Wavelets of each scale are slid along the time series to assess their degree of correspondence at each time point using a mathematic procedure known as convolution. Then a calculation is made to determine whether the smaller- or the larger-scale wavelets are more dominant in the data by looking at the values of the convolution for all scales. Predominance of longer scales is indicative of persistence, whereas predominance of shorter scales indicates antipersistence. The original WTMM approach provides one measure of persistence, using both upward- and downwardpointing wavelets. However, in this study, measures of persistence were calculated separately for low-high-low and high-low-high activity patterns. 
Ohashi et al.: Locomotor Microactivities Associated with Therapeutic Responses in

Fig. 1. Locations of modulus minima and maxima, and the average, variance, and DFA exponent of locomotor activities. a A daytime record of locomotor activity collected by a wrist-worn actigraph in a patient with SAD. b Integrated physical activity. c Locations of local minima of the wavelet transform of the integrated series. d Location of the local maxima. The locomotor activities (a) and the integrated activities (b) are colored red at the locations of all the minima and blue for all the maxima, based on wavelet time frames of about 26 and $40 \mathrm{~min}$. The data are colored yellow when the minima and maxima overlap. Green curves on the actigraph records show the Gaussian 2nd derivative with the local linear trend, and those on the integrated activities show the Gaussian 3rd derivative with the 2nd-order polynomial trend detecting one local minimum (left) and maximum (right) at their locations.

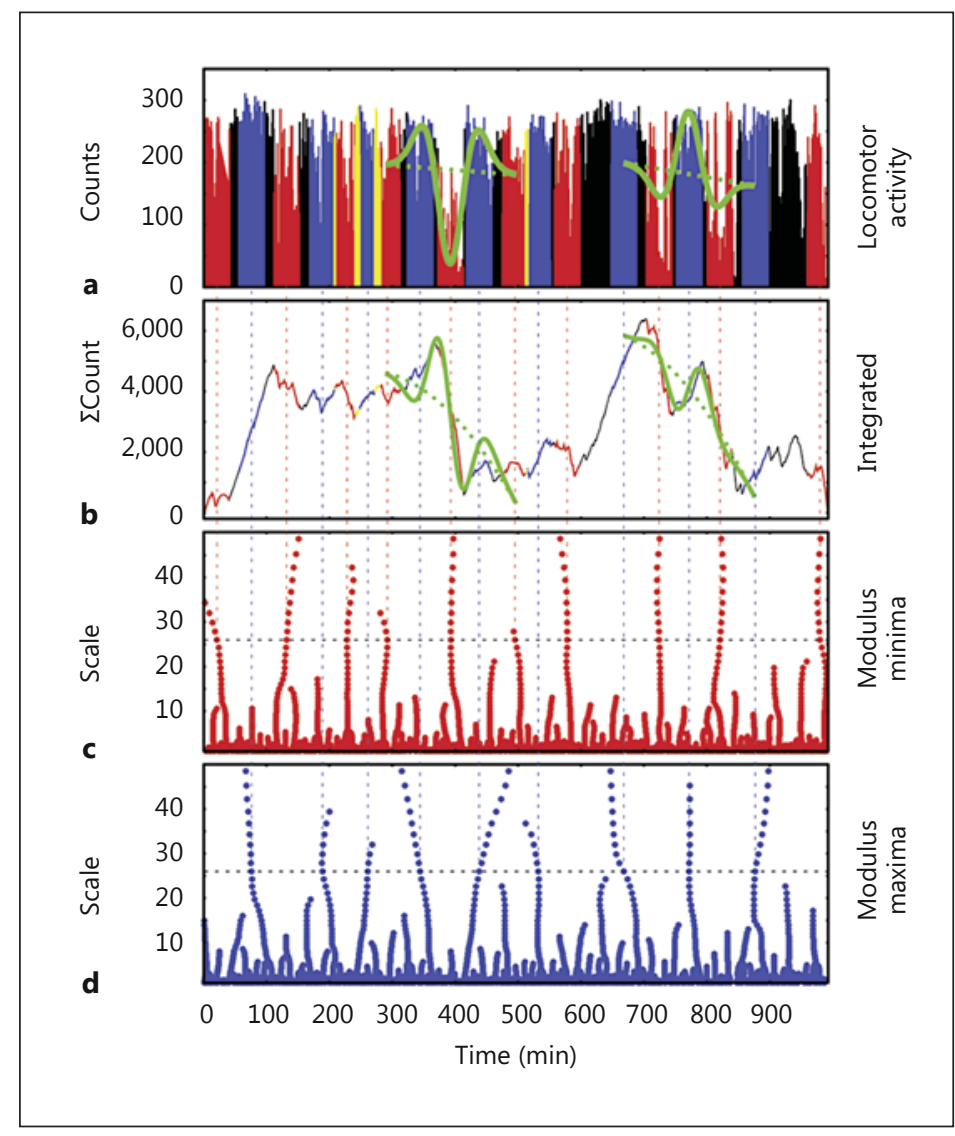

The more rigid procedure of the analysis is as follows. In this study, we used the Gaussian 3rd derivative

$$
\psi(t)=t\left(3-t^{2}\right) e^{-0.5 t^{2}}
$$

as a mother wavelet to examine the integrated locomotor activity data. This is equivalent to using the Gaussian 2nd derivative (so-called 'Mexican hat') wavelet to examine the raw signals though the integrated approach automatically removes the local mean and the local linear trend (fig. 1b).

In the WTMM method [16], the degree of the temporal correlation is calculated from a partition function $Z_{q}(a)$, defined as the sum of the $q$-th powers of the local maxima of the modulus of the wavelet transform coefficients at wavelet scale $a$ from the entire record. The moment parameter $q$ is used to control the degree of emphasis on the magnitude of fluctuations matching the wavelet template; for example, if $q=0, Z_{q}(a)$ just counts the number of maxima/minima at the scale $a$, irrespective of whether they are large or small in terms of the magnitude. In case of $q=2$, however, the average degree of predominance of fluctuation at the scale $a$ is evaluated by the squared local maxima, i.e., the variance, at that scale. With $q \geq 2$, large fluctuations matching the template (e.g., large troughs in fig. 5) predominantly determine the results. The power-law scaling of $Z_{q}(a)$, or a linear relationship between $\log (a)$ and $\log \left(Z_{q}(a)\right.$ ), yields the scaling exponents $\tau(q)$ for small $a$. The scaling exponents $\tau(q)$ is then related to the so-called 'local Hurst exponent' by $h \approx \Delta \tau(q) / \Delta q$ [17], which quantifies the overall degree of temporal correlation of the time series. 
Ohashi et al.: Locomotor Microactivities Associated with Therapeutic Responses in Patients with Seasonal Affective Disorders

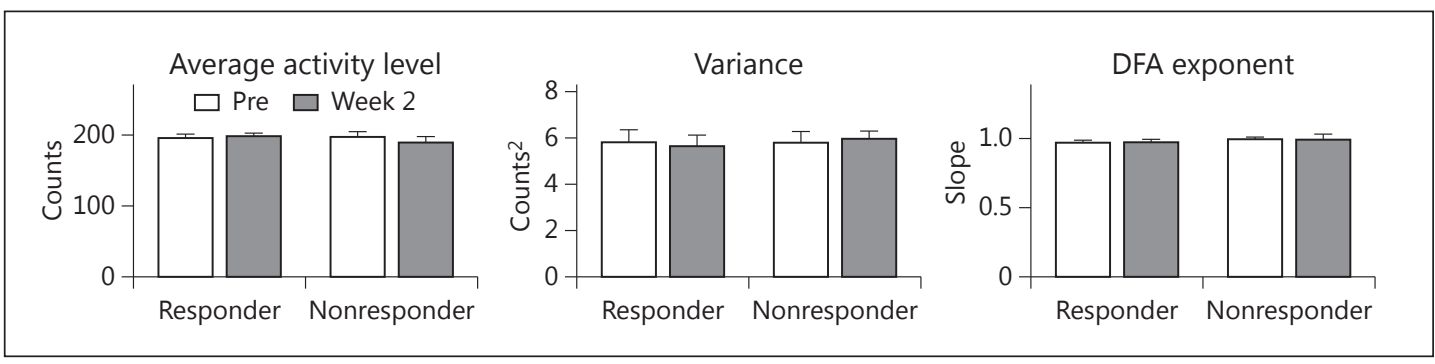

Fig. 2. Measures of average activity, variance, and global scaling. Differences between light therapy responders versus nonresponders in average activity counts per minute, the average within-subject variance, and the global scaling exponent calculated by the DFA [18]. Vertical bars represent SEM. There were no significant differences in any of the values between responders and nonresponders. Pre $=$ Before treatment.

Ohashi et al. [6] reported that, in many real-world signals including locomotor activity time series, the time correlation is asymmetrical between positive and negative changes suggesting different scale-invariant dynamics depending on the direction of the changes. In particular, in locomotor activity, the power-law scaling exponent at the higher levels of activity (corresponding to local maxima of the wavelet transform coefficients) and that at the lower levels of activity (corresponding to local minima) would correspond to qualitatively different physiological states, i.e., active and rest, respectively. Thus, in this study, we analyzed the time correlation for local maxima and minima separately by calculating the partition functions $Z_{q}{ }^{M}(a)$ and $Z_{q}{ }^{m}(a)$ from the maxima and the minima of the wavelet coefficients, respectively $[6,8]$.

The partition functions were averaged over all the days prior to therapy and all the days during the second week of therapy to construct $Z_{q}{ }^{M}(a)$ and $Z_{q}{ }^{m}(a)$ for each patient. The scaling exponents $\tau(q)$ were obtained for the average of $0.97<\log (a) \leq 1.74$, which correspond to about 9-55 min. As shown in figure 1, the minima (maxima) identify periods with lower (higher) levels of activity than the surrounding periods. The exponents $\tau(q)$ and $h \approx \Delta \tau(q) / \Delta q$ were calculated for $0 \leq q \leq 3$, where asymmetrical properties were observed for locomotor activity time series of healthy individuals [6].

Before conducting the wavelet analysis, we calculated the average, the variance, and the global scaling exponent - calculated by the detrended fluctuation analysis (DFA) [18] - of the activity time series. None of these parameters differed significantly between responders and nonresponders (fig. 2), giving rise to the need for a measure of local dynamics to better delineate the effects of treatment on psychomotor activity.

\section{Statistical Analysis}

The average $h \approx \Delta \tau(q) / \Delta q$ for $0 \leq q \leq 3$ was calculated separately for the responders and nonresponders before and during the therapy. A repeated-measures analysis of variance (ANOVA) was performed using SAS software (SAS Institute, Cary, N.C., USA) to evaluate the effects of therapy, group (responders or nonresponders), and direction (maxima or minima) on the average $h$, with the subject effects nested in the group as a factor.

\section{Results}

Prior to treatment, responders and nonresponders had mean SIGH-SAD scores of 29 [95\% confidence intervals (CI), 24-34] and 28 (95\% CI, 23-33), respectively $[\mathrm{F}(1,16)=$ 0.063 , p > 0.8]. During treatment, SIGH-SAD scores fell by $76 \%(95 \% \mathrm{CI}, 60-92)$ in responders 


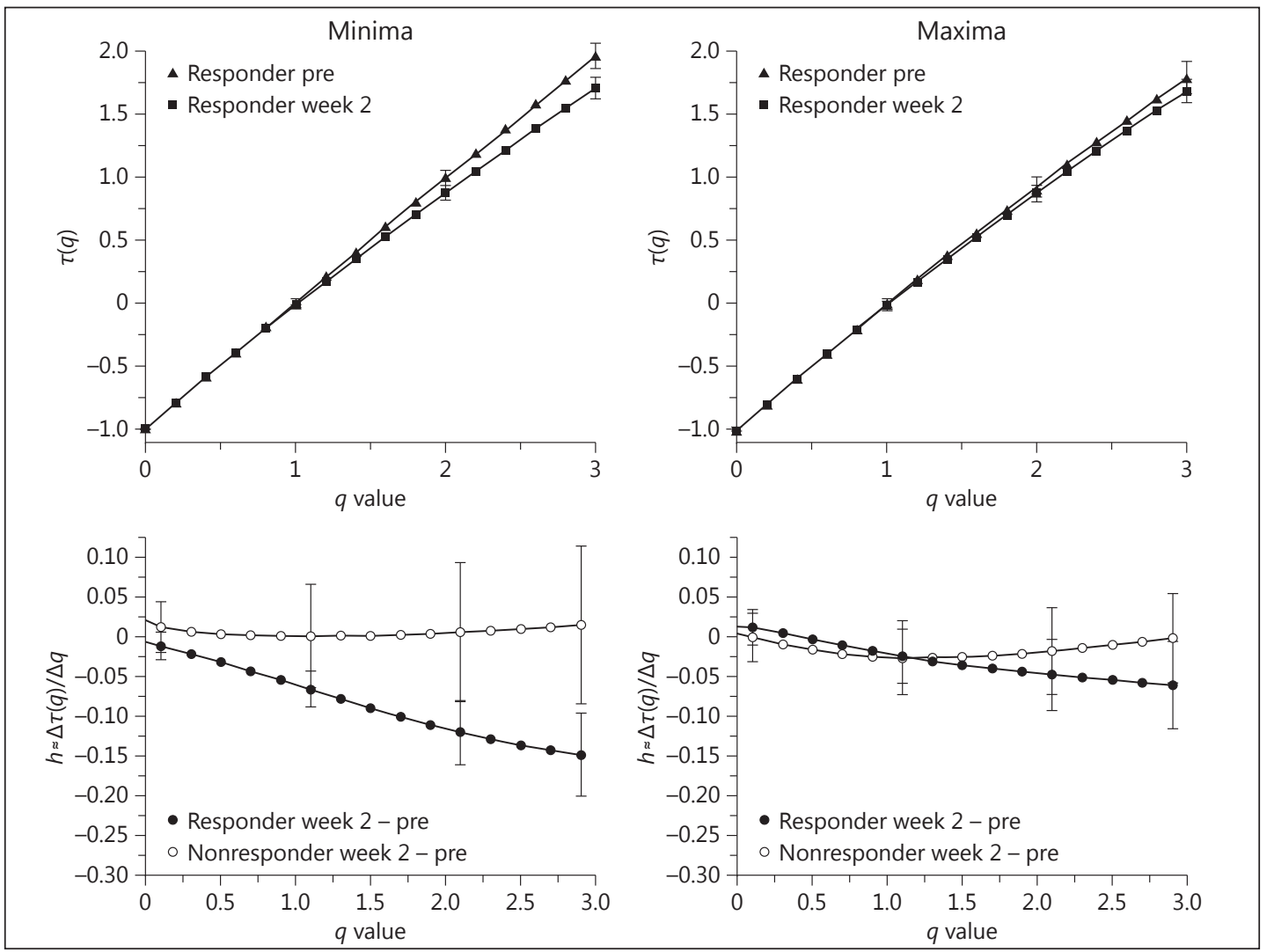

Fig. 3. Multifractal spectra and Hurst exponents. Multifractal $\tau(q)$ spectra for responders prior to light treatment and during the second week of phototherapy within the range of $0 \leq q \leq 3$ (top), where $q$ is the moment parameter. Comparison between responders and nonresponders (bottom) showing the changes in Hurst exponent $h$ within the range $0 \leq q \leq 3$. The multifractal spectra of nonresponders were similar before and during the therapy for minima and maxima (data not shown). The vertical bars represent SEM for each group. Pre $=$ Before treatment.

and by only $17 \%$ (95\% CI, 3.7-31) in nonresponders, which was a direct consequence of the way the groups were defined.

The results of the repeated-measures ANOVA showed no significant main effects of group, direction, or phototherapy for the average $h$ for $0 \leq q \leq 3$. However, there was a significant 'group $\times$ therapy' interaction $[F(1,27)=7.47, p=0.01]$ indicating that the responders and nonresponders reacted differently to phototherapy. Thus, for each group, an additional repeated-measures ANOVA was conducted to examine the effects of therapy and minimamaxima direction with subjects included as an independent factor. The results revealed that the effect of the therapy was significant only in the responder group $[F(1,7)=8.34, p=0.02$; nonresponders: $\mathrm{F}(1,7)=0.04, \mathrm{p}>0.8$ ] . The local Hurst exponent $h$ in responders fell from 0.983 (95\% CI, 0.905-1.061) to 0.898 (95\% CI, 0.833-0.963). In contrast, $h$ for nonresponders was $0.916(95 \% \mathrm{CI}, 0.777-1.055)$ at baseline and 0.923 (95\% CI, 0.830-1.016) during the second treatment week.

Post hoc paired t tests showed that significant alterations in $h$ for responders occurred during the minima [ $\mathrm{t}(7)=3.25, \mathrm{p}<0.02]$ but not the maxima [ $\mathrm{t}(7)=0.83, \mathrm{p}>0.4]$.

This can be seen in the $\tau(q)$ spectra for the minima (fig. 3, top). The slope of $\tau^{m}(q)$, which is equivalent to the local Hurst exponent $h$ for $0 \leq q \leq 3$, was smaller for the second week of 
Fig. 4. $\tau(2)$ changes in responders and nonresponders. The $\tau(q)$ value at $q=2$ for each subject in the responder group (top; filled circles) and the nonresponder group (bottom; open circles) before and during the second week of phototherapy. The average and the SEM are shown in the left and the right of each period for both groups. The patients in the responder group showed significant decreases in the $\tau(2)$ values, while the changes in the nonresponder group were less consistent. Pre $=$ Before treatment.

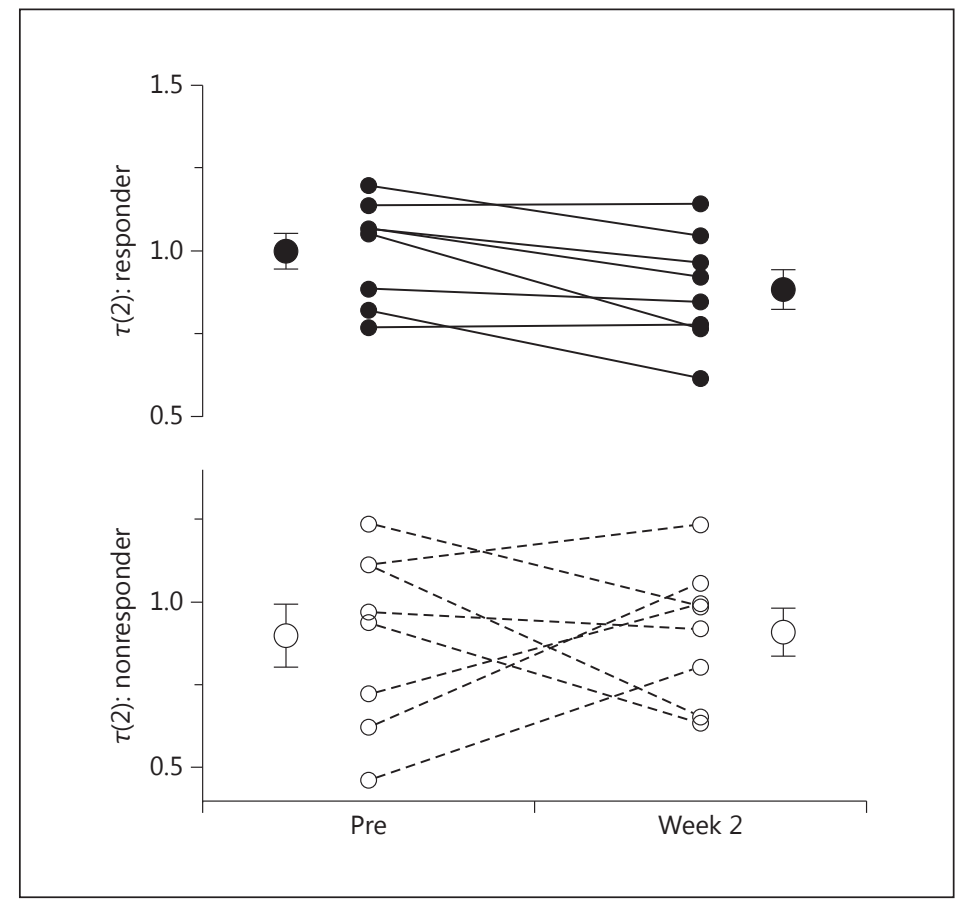

therapy compared to the pretreatment baseline in the responder group (fig. 3, top left). In addition, the value of $\tau(2)$ for the minima, which is equivalent to the DFA exponent but calculated by using the minima only, was decreased during therapy in the responder group [t(7) = 3.148, $\mathrm{p}<0.02$ ], while there was no significant difference in the nonresponder group (fig. 4). The difference between $h \approx \Delta \tau(q) / \Delta q$ prior to and following treatment in each group (fig. 3 , bottom) shows that for the minima the responders' $h$ was decreased during therapy, particularly for larger $q$, while there was no significant change observed in the nonresponder group. This indicates that the responders had significantly smaller $h$ or stronger antipersistence at lower levels, or larger troughs, of activity after the initiation of treatment. This difference was not seen in nonresponders. In neither group were there significant differences in persistence before and after light therapy during periods with higher levels of the activity.

In responders, locomotor activity before therapy was characterized by having slower or 'sluggish' transitions at the lower levels of activity, and the pattern was altered during treatment to show more rapid transitions. In other words, the activity pattern of responders prior to treatment was characterized by the presence of epochs of low-level activity which were persistently followed by further periods of low-level activity. During the second week of treatment, this pattern changed, i.e., during epochs of relative inactivity, there was a less persistent tendency to remain at low levels of activity.

Figure 5 shows the typical 3-day recordings of the locomotor activity in a patient in the responder group. During the pretreatment period (fig. 5a), the wavelet transform coefficient for the minima had greater values at larger scales, which are colored in red-yellow. In contrast, during the posttreatment period (fig. 5b), there was a decrease in these episodes. Greater wavelet transform coefficients generally appear in the graph as broad epochs in which minute-to-minute measures of activity fall to relatively low levels (fig. 5a). After treatment, minima epochs tend to be less broad and/or less deep.

The change in the responders' locomotor activity from slower transitions at the lower levels of activity to more rapid transitions was accompanied by an improvement in the SIGH-SAD item score of psychomotor retardation based on the observation of the interviewer 
Fig. 5. Locomotor activity and wavelet transform coefficient before and at the 2 nd week of phototherapy. Typical example of 3-day locomotor activity (top) and the values of the wavelet transform coefficient (bottom) for the minima from patients categorized as responders before (a) and during (b) the 2nd week of phototherapy. The 2 nd and the 3 rd days are shifted so that they start from 1,000 and 2,000 min, respectively. The wavelet transform coefficients are colored according to their values for those larger than 2,000 (right bar). Values $<2,000$ are colored gray. Slower drops with the lower activity levels are seen in the pretherapy locomotor activity where the wavelet transform coefficient has larger values (yellow-orange). The drops are much more rapid in the 2 nd week of therapy, and the minima have the smaller wavelet transform coefficients at larger scales.

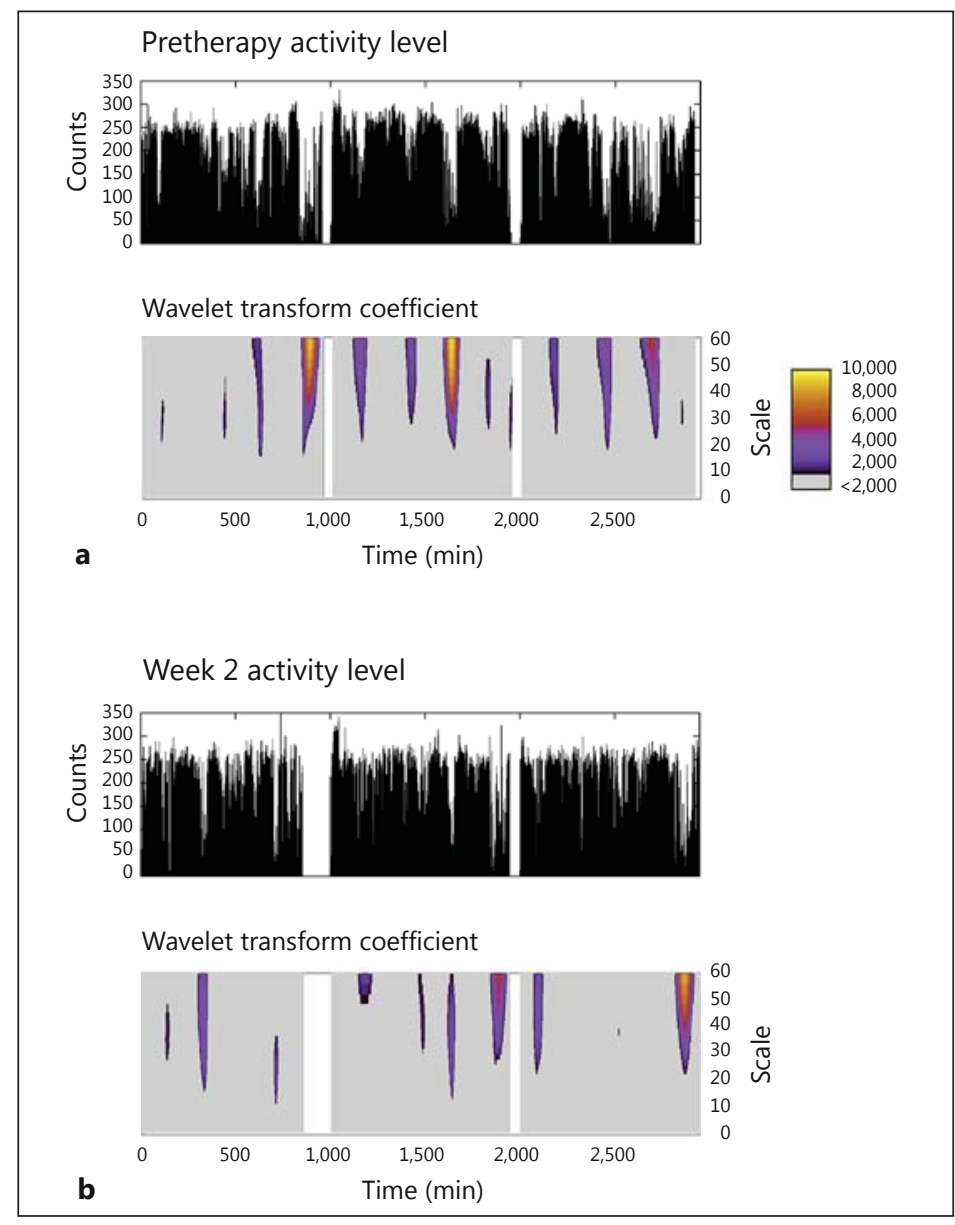

(table 1). Before treatment, the scores were comparable between responders and nonresponders (Wilcoxon rank sum test; $p>0.23$ ). However, after treatment, there were significant differences in the scores between the two groups $(\mathrm{p}<0.008)$, and the responders showed marked decreases in this score.

Light therapy responders reporting higher fatigability had more 'sluggish' bursts in activities and higher values of the maxima-to-minima ratio of $h$ before treatment. There was a highly significant correlation between the SIGH-SAD item score of fatigability and the maxima-tominima ratio $(r=0.926, p<0.001)$ in responders (fig. 6). A significant correlation was also seen between SIGH-SAD scores for item number 15, which asks about loss of energy and fatigability, and the maxima-to-minima ratio $(\mathrm{r}=0.900, \mathrm{p}<0.003)$ in this group. These two items contributed to the significant correlation between the total SIGH-SAD scores and the maxima-to-minima ratio $(r=0.961, p<0.0002)$ in responders before treatment. Neither of these correlations were found in the nonresponder group before treatment (maxima/minima vs. fatigability, $r=0.427$, $p=0.291$; loss of energy, $r=-0.374, p=0.361$; total SIGH-SAD, $r=0.634, p=0.091$ ).

\section{Discussion}

SAD patients who were responsive to light therapy showed a significant increase in antipersistence at the lower levels of activity. We postulate that persistence during periods of rest or relative inactivity may be an indication of psychomotor retardation, and the acceleration of 


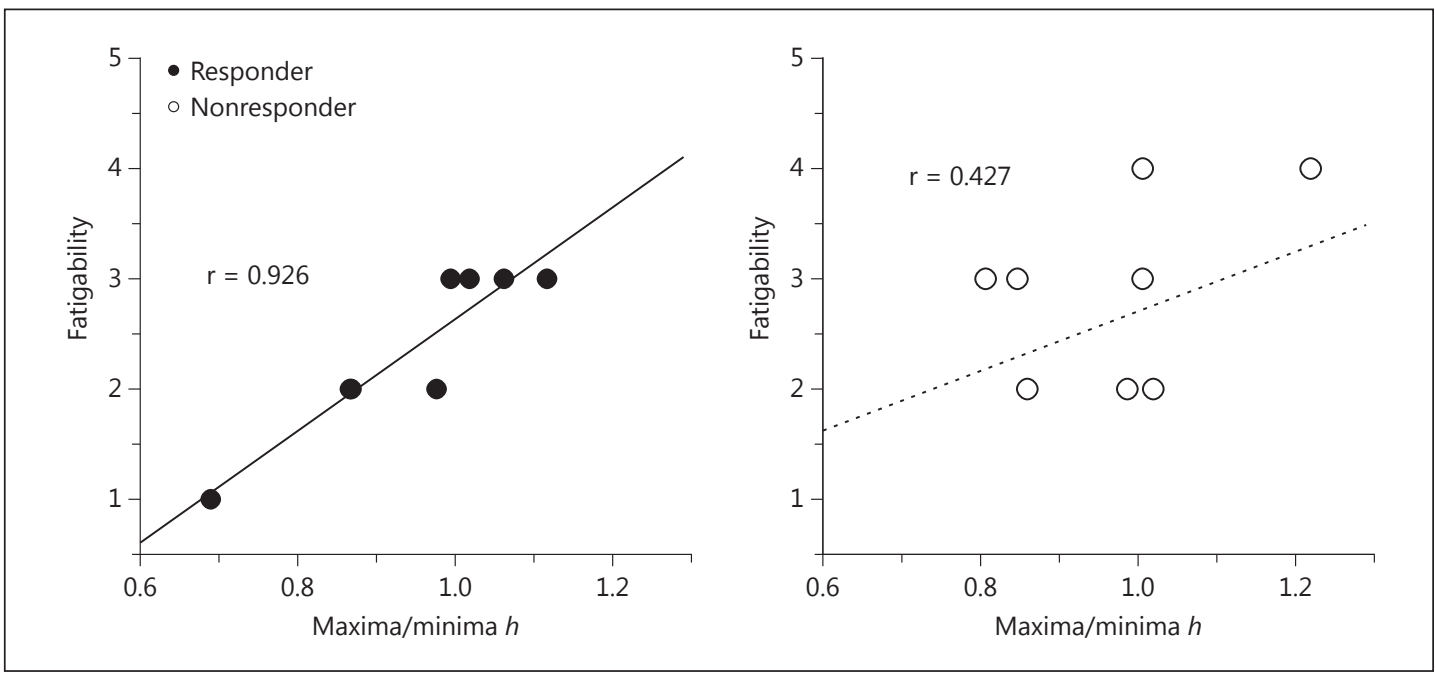

Fig. 6. Relation between fatigability and the scaling exponents. The relation between the ratio of the scaling exponents (maxima/minima) and the SIGH-SAD item score of fatigability for patients in the responder group before treatment $(r=0.926, p<0.001$; regression line: $y=5.0708 x-2.4367)$.

these transitions may indicate an alleviation of this sign of depression. The changes due to therapy were discernable only when we separately analyzed the time correlation at the higher and lower levels of activity, as the therapeutic effect was seen only at the lower levels of activity. This suggests that the global scaling exponents calculated by conventional fluctuation analyses that evaluate the time correlation of the entire record would obscure the changes due to the therapy. In locomotor activity time series, the time correlation at different levels of activity corresponds to substantially different phases of patients' activity, i.e., active and rest. Hence, it seems important to analyze the time correlation at higher and lower levels of activity separately.

In treatment-responsive SAD patients, baseline ratios of the exponents at maxima versus minima were significantly correlated with the ratings of fatigability $(r=0.926)$ as well as with the total SIGH-SAD score $(r=0.961)$. Thus, in the responsive patients before treatment, the severity of their symptoms seemed to be correlated with the persistence during higher levels of activity compared to lower levels of activity, which is consistent with the result for chronic fatigue syndrome patients [6]. These correlations were not significant in the nonresponder group. It is interesting to speculate that phototherapy-responsive SAD patients show a close match between subjective symptom and the microstructure of their activity. In contrast, there appears to be a mismatch between subjective state and objective measures in nonresponders.

\section{Conclusions}

Overall, this study reveals differences between responders and nonresponders to phototherapy in the microstructure of their rest-activity rhythms. Effective treatment appears to exert discernible effects in the microstructure on the way activity fluctuates during periods of reduced activity. Wavelet analysis may have revealed a specific signature reflecting periods of psychomotor retardation and their abatement during treatment. This approach is far more sophisticated and more revealing than traditional measures of mean activity. Further research is needed, however, to establish a connection between the antipersistence at lower levels of activity and psychomotor retardation. Few studies have performed microstructure analysis 
of rest-activity rhythms in patients with psychiatric disorders. One preliminary report suggests the possibility that activity time series of some manic-depressive patients may show temporal power-law correlations [4]. More recent papers have shown altered scaling properties in major depression using a threshold to distinguish low versus high activity periods [19] and an altered distribution of wavelet amplitudes in subjects with bipolar disorders [20]. The present findings suggest that it may be fruitful to utilize this approach in other patient populations to assess the effects of psychotropic medications on the parameters as well as the correlations between these parameters and ratings of symptom severity.

\section{Acknowledgements}

We thank Ann Polcari, PhD, RN, Carol A. Glod, RN, CS, PhD, and Cynthia E. McGreenery for recruiting subjects and collecting the data, and Z.R. Struzik, PhD, for the discussions and helpful suggestions. This study was supported by the US NIMH award R01-MH48343 to M.H.T.

\section{References}

$>1$ Rosenthal NE, Sack DA, Gillin JC, Lewy AJ, Goodwin FK, Davenport Y, et al: Seasonal affective disorder. A description of the syndrome and preliminary findings with light therapy. Arch Gen Psychiatry 1984;41:72-80.

-2 Teicher MH, Glod CA, Magnus E, Harper D, Benson G, Krueger K, et al: Circadian rest-activity disturbances in seasonal affective disorder. Arch Gen Psychiatry 1997;54:124-130.

-3 Teicher MH: Actigraphy and motion analysis: new tools for psychiatry. Harv Rev Psychiatry 1995;3:18-35.

-4 Selz KA, Mandell AJ, Anderson CM: Distributions of local Mandelbrot-Hurst exponents: motor activity in fetal rats of cocainized mothers and manic-depressive patients. Fractals 1995;3:893.

5 Ohashi K, Polcari A, Teicher MH: Scale-invariant locomotor activity patterns in children with SAD. Int J Integr Med 2013;1:1.

6 Ohashi K, Nunes Amaral LA, Natelson BH, Yamamoto Y: Asymmetrical singularities in real-world signals. Phys Rev E Stat Nonlin Soft Matter Phys 2003;68:065204.

7 Fukuda K, Straus SE, Hickie I, Sharpe MC, Dobbins JG, Komaroff A: The chronic fatigue syndrome: a comprehensive approach to its definition and study. International Chronic Fatigue Syndrome Study Group. Ann Intern Med 1994;121:953-959.

8 Ohashi K, Bleijenberg G, van der Werf S, Prins J, Amaral LAN, Natelson BH, et al: Decreased fractal correlation in diurnal physical activity in chronic fatigue syndrome. Methods Inf Med 2004;43:26-29.

-9 Pan W, Ohashi K, Yamamoto Y, Kwak S: Power-law temporal autocorrelation of activity reflects severity of parkinsonism. Mov Disord 2007;22:1308-1313.

10 Pan W, Soma R, Kwak S, Yamamoto Y: Improvement of motor functions by noisy vestibular stimulation in central neurodegenerative disorders. J Neurol 2008;255:1657-1661.

-11 Terman JS, Terman M, Schlager D, Rafferty B, Rosofsky M, Link MJ, et al: Efficacy of brief, intense light exposure for treatment of winter depression. Psychopharmacol Bull 1990;26:3-11.

12 Winkler D, Pjrek E, Praschak-Rieder N, Willeit M, Pezawas L, Konstantinidis A, et al: Actigraphy in patients with seasonal affective disorder and healthy control subjects treated with light therapy. Biol Psychiatry 2005; $58: 331-336$.

13 Williams J, Link MJ, Rosenthal NE, Amira L: Structured Interview Guide for the Hamilton Depression ScaleSeasonal Affective Disorder Version (SIGH-SAD). New York, New York State Psychiatric Institute, 1992.

14 Spitzer RL, Gibbon M, Williams JBW: User's Guide for the Structured Clinical Interview for DSM-IV Axis I Disorders (SCID-I). Washington, American Psychiatric Press, 1997.

15 Cole RJ, Kripke DF, Gruen W, Mullaney DJ, Gillin JC: Automatic sleep/wake identification from wrist activity. Sleep 1992;15:461-469.

16 Muzy JF, Bacry E, Arneodo A: The multifractal formalism revisited with wavelets. Int J Bifurcat Chaos 1994, DOI: $10.1142 / \mathrm{S} 0218127494000204$.

17 Vicsek T: Fractal Growth Phenomena. Singapore, World Scientific, 1989.

18 Peng CK, Havlin S, Stanley HE, Goldberger AL: Quantification of scaling exponents and crossover phenomena in nonstationary heartbeat time series. Chaos 1995;5:82-87.

19 Nakamura T, Kiyono K, Yoshiuchi K, Nakahara R, Struzik ZR, Yamamoto Y: Universal scaling law in human behavioral organization. Phys Rev Lett 2007;99:138103.

20 Indic P, Salvatore P, Maggini C, Ghidini S, Ferraro G, Baldessarini RJ, et al: Scaling behavior of human locomotor activity amplitude: association with bipolar disorder. PLoS One 2011;6:e20650. 